\title{
Convulsive syncope related to a small dose of quetiapine in an adolescent with bipolar disorder
}

\author{
This article was published in the following Dove Press journal: \\ Neuropsychiatric Disease and Treatment \\ 19 July 2017 \\ Number of times this article has been viewed
}

\author{
Jianbo Lai ${ }^{1,2}$ \\ Qiaoqiao $\mathrm{Lu}^{3}$ \\ Tingting Huang ${ }^{3}$ \\ Shaohua $\mathrm{Hu}^{1,2}$ \\ Yi Xu ${ }^{1,2}$
}

'Department of Psychiatry, First Affiliated Hospital, Zhejiang University School of Medicine, ${ }^{2}$ Key Laboratory of Mental Disorder Management in Zhejiang Province, ${ }^{3}$ Department of Internal Medicine, Zhejiang University School of Medicine, Hangzhou, China
Correspondence: Shaohua Hu; Yi Xu Department of Psychiatry, First Affiliated Hospital, Zhejiang University School of Medicine, Key Laboratory of Mental Disorder Management, 79 Qingchun Road, Hangzhou, Zhejiang 310003, China Tel +86 $57 \mid 56723002 ; 13805746579$ Fax +86 57| 5672300 I Email dorhushaohua@zju.edu.cn; xuyizju6I@I63.com

\begin{abstract}
Quetiapine, an atypical antipsychotic, has been extensively used in patients with bipolar disorder. Overdose of quetiapine can result in severe complications, such as coma, seizure, respiratory depression, arrhythmia, and even death. However, the paucity of toxicological evaluation in adolescence causes more potential risks in this population. Herein, we present a case of hypotension and convulsive syncope after exposure to a small dose of quetiapine in a 16-year-old who was diagnosed with bipolar disorder. After cessation of quetiapine, no additional convulsive movements were reported. This case indicates that even in young patients without predisposing factors, close monitoring of adverse effects should be warranted for safety concerns, especially at the initiation of quetiapine treatment.
\end{abstract}

Keywords: quetiapine, bipolar disorder, hypotension, convulsive syncope

\section{Introduction}

Quetiapine, a second-generation antipsychotic, is approved by US Food and Drug Administration to treat young patients (age $\geq 10$ years) with acute depressive or manic episodes and maintenance of bipolar disorder. ${ }^{1}$ Quetiapine seems to be a promising agent, well tolerated in the short and medium term in children and adolescents, and has low potential to elicit extrapyramidal effects. ${ }^{2}$ However, extremely limited guidelines for its use are available in this population. A recent nationwide report demonstrated that off-label use of quetiapine in children and adolescents is prevalent in Denmark, and life-threatening adverse events involved seizure, QT-interval prolongation, and cerebral hemorrhage. ${ }^{3}$ Besides, this study implied that quetiapine could induce hyperprolactinemia, especially in minors. ${ }^{3}$ Moreover, in some susceptible individuals, exposure to quetiapine overdoses can result in severe complications, such as coma, seizure, respiratory depression, arrhythmia, and even death. ${ }^{4}$ Therefore, great care should be taken when initiating quetiapine treatment in young patients.

For example, new-onset seizure has been reported in two young patients after taking small doses of quetiapine. ${ }^{5}$ One of these two patients was a 16-year-old girl with ornithine transcarbamylase deficiency and mental retardation, who was prescribed quetiapine to manage her behavioral instability. She developed a generalized tonicclonic seizure after ingesting $50 \mathrm{mg}$ quetiapine for 2 consecutive days. ${ }^{5}$ Another was a 7-year-old boy with autism and mental retardation, who was prescribed quetiapine at a dose of $25 \mathrm{mg} /$ day for his behavioral problems. Generalized tonic-clonic seizure occurred as early as the first time quetiapine was taken. ${ }^{5}$ According to the literature available, most cases of seizure secondary to quetiapine exposure occur in adult patients or present as acute intoxication because of overdoses. ${ }^{6-8}$ Evidence of severe adverse events associated with quetiapine treatment in children and adolescents is fairly rare. 
Herein, we depict an adolescent with bipolar disorder who developed hypotension and syncope with convulsive movements after ingestion of quetiapine without concomitant medications. This case enriches our knowledge of quetiapine toxicity in young people.

\section{Case presentation}

A 16-year-old high school teenager was admitted to our hospital because of episodic mood swings for almost 4 years. Periods of depression were predominant in his course of disease and lasted for 1 or 2 months. When depressed, this patient complained of waning interest, poor academic performance, bad memory, and loss of appetite. Sometimes, this patient would have suicidal ideations, and had once attempted suicide by holding his breath. He had auditory hallucinations and delusions of persecution. Comparatively, when his mood elevated, this patient would become energetic, conversant, and generous, and gradually became euthymic after nearly 1 week. Two weeks before admission, this patient was sent to the outpatient service by his family. No physical illness, epilepsy, history of head trauma, or family history of mental disorders was reported. He had lost his mother because of a traffic accident. He denied experience of smoking, drinking alcohol, or taking any toxic or illicit substance. Neurological examination was normal, as well as laboratory tests (eg, routine blood test, urine, thyroid hormones, reproductive hormones, biochemical indices, and infectious profiles). Magnetic resonance imaging of the brain indicated an enlarged left lateral ventricle (Figure 1). According to the

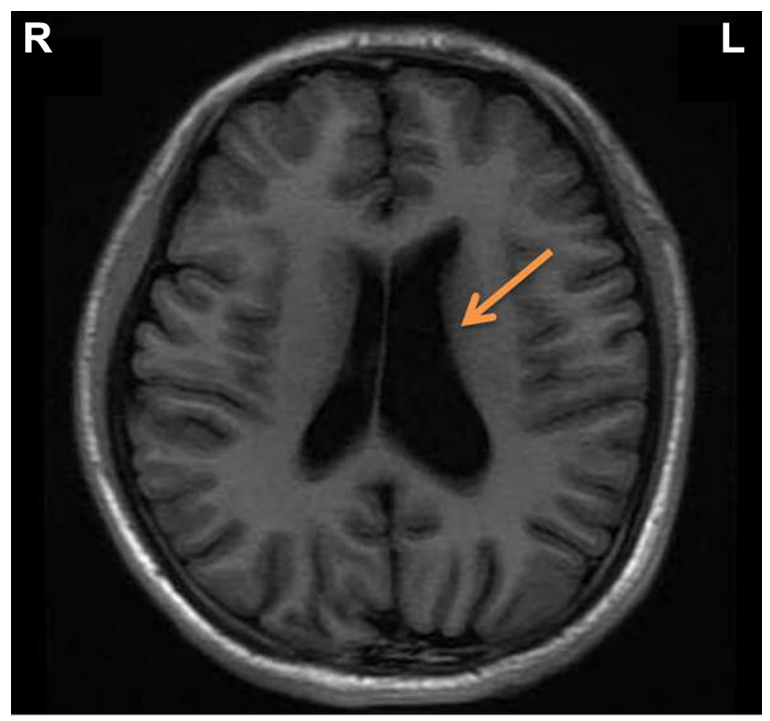

Figure I Magnetic resonance imaging showing an enlarged left lateral ventricle (orange arrow).
Diagnostic and Statistical Manual of Mental Disorders (fifth edition), this patient matched with the diagnosis of bipolar II disorder and depressive episodes, with psychotic features. Consequently, quetiapine was prescribed and started at a dosage of $50 \mathrm{mg}$ per night. However, when this patient took the first $50 \mathrm{mg}$, he slept for almost 12 hours and felt feeble in the daytime. Since then, he had decided to stop this medication. His depressive condition did not improve, and hospitalization was suggested by his psychiatrist.

On admission, all vital signs of this patient were normal. His grandmother stayed in hospital to look after him. This patient tried to take an afternoon nap, but failed to fall asleep, and was given $50 \mathrm{mg}$ quetiapine as a sleeping aid by his grandmother. About 2 hours after quetiapine exposure, this patient was taken to the nurses' desk and educated with notes on security as a new inpatient. All of a sudden, his complexion became pale. Immediate blood glucose was $5.5 \mathrm{mmol} / \mathrm{L}$, heart rate was 72 beats per minute, and blood pressure $118 / 80 \mathrm{mmHg}$. The psychiatrist arrived quickly and tried to measure blood pressure again, but failed to get any result. On his way back to the ward when supported by others, this patient fell abruptly to the floor, followed by tics of limbs and opisthotonos, which lasted for 2-3 seconds, and lay on the floor unconscious. No foaming at the mouth, urinary incontinence, or tongue biting was observed. About 1 minute later, he stood up by himself and asked, "What happened just now? My mind went blank." At this moment, his complexion gradually became fresh and rosy. Blood pressure was at $88 / 53 \mathrm{mmHg}$, and heart rate was at 61 beats per minute. As the patient's condition had improved, he was given low-flow oxygen therapy with close electrocardiography monitoring. Serum creatinine kinase after this event was 1,960 units/L (reference range 38-174 units/L). We did not examine the quetiapine concentration in the blood, as the patient had taken a small dosage of this medication. He was told not to take this medication again. A combination of valproate and olanzapine was used as his therapeutic regimen. Electroencephalography (EEG) was conducted 5 days after the event, and no epileptiform activity was captured. This patient recovered well in the next 2 weeks and remained syncope-free before hospital discharge. During outpatient visits, no more convulsive syncope was reported.

The Institute Ethical Committee of the First Affiliated Hospital, Zhejiang University School of Medicine approved this case study. Written informed consent for publication of the case and any accompanying image was obtained from the patient and his guardian. 


\section{Discussion}

In this study, we present a case of extreme hypotension, transient convulsive movements, and unconsciousness after quetiapine exposure, suggesting relevant adverse effects to a small dosage of quetiapine $(50 \mathrm{mg})$ in an adolescent with bipolar disorder. No predisposing factors were identified in this patient. To our knowledge, this is the first case study suggesting convulsive syncope secondary to a small dosage of quetiapine $(50 \mathrm{mg}$ ) in an adolescent with bipolar disorder.

Quetiapine functions as a multireceptor antipsychotic involved in the dopamine, serotonin, and adrenergic pathways. It also has antihistamine and anticholinergic properties. Hypotension is known as a common adverse effect, which is considered to be elicited by inhibition of adrenergic $\alpha_{1}$-receptors. ${ }^{9}$ However, the mechanism behind quetiapineinduced seizure remains unclear. Structurally similar to quetiapine, the dose-dependent potential of clozapine to mediate seizure by lowering the threshold of seizure is more definite. ${ }^{10}$ The risk of seizure provocation induced by first-generation antipsychotics or second-generation antipsychotics is inconsistent across different studies. In a nest-control study, when applied to patients with affective disorders, medium- to highpotency first-generation antipsychotics were more likely to elicit seizure than all other antipsychotics. ${ }^{11}$ Nevertheless, other findings suggested second-generation antipsychotics, rather than first-generation antipsychotics, had a higher risk of seizure. ${ }^{12}$ In addition, abnormal alterations on EEG have been observed in psychiatric patients taking secondgeneration antipsychotics, which were particularly high under clozapine and olanzapine treatment. ${ }^{13}$ None of the patients with quetiapine treatment showed EEG abnormalities in the same study. ${ }^{13}$ Interestingly, in a rat model with malformations of cortical development, quetiapine was found to rescue its cognitive impairment and reduce seizure susceptibility, possibly by maintaining the integrity of myelin. ${ }^{14}$

In our patient, we noted that extremely low blood pressure was associated with convulsive movements. In individuals with quetiapine abuse, severe complications, such as hypotension, respiratory depression, and seizure, have also been observed. ${ }^{15}$ Unfortunately, the interrelationship between hypotension and seizure has always been neglected. In a rare case of seizure presenting as refractory hypotension, the authors provided sufficient evidence to support acute hypotension as an atypical manifestation of seizure. ${ }^{16}$ Hypotension during the onset of seizure may cause cerebral hypoperfusion and further worsen autonomic dysfunction. ${ }^{16}$
Of note, we would like to differentiate the manifestations of convulsive syncope from those of seizure attacks. In our patient, acute onset of limb spasm, opisthotonos, and transient unconsciousness was observed without other typical symptoms of seizure, such as foaming at the mouth, loss of urine, or biting of the tongue. Therefore, the seizure-like movements in our patient were not typical, and were more likely to be a syncopal attack secondary to hypotension. Convulsive syncope, or in academic terms, reflex asystolic syncope, occurs predominantly in young people and can manifest as loss of consciousness, loss of postural tone, myoclonic movements, and nonepileptic spasms. ${ }^{17}$

In the present case study of a patient with bipolar disorder, hypotension associated with convulsive movements was observed nearly 2 hours after exposure to a small dose of quetiapine. This is in accordance with the serum peak time after taking quetiapine orally. ${ }^{18}$ No obvious predisposing factors were reported, and no other medications were coingested. However, the undetected quetiapine concentration in blood seemed to be a major limitation of our study. In individuals with specific polymorphisms in CYP3A4 enzymes, unpredictable adverse reactions were associated with decreased enzyme activity and increased blood concentration. ${ }^{19}$ Moreover, no additional convulsive movements were observed after discontinuing quetiapine treatment. According to a scale evaluating possible drugrelated adverse effects, the relationship between reported adverse reactions and quetiapine in our patient was assigned to the "probable" category. ${ }^{20}$ Finally, we hypothesize that the convulsive movements were consequences of hypotension induced by quetiapine.

In conclusion, this single case report enriches our understanding of quetiapine-associated adverse reactions in young patients. Special concerns are essential to evaluate the risk of hypotension and convulsive syncope in children and adolescents before starting quetiapine treatment. Continuous monitoring of acute adverse events is also needed after the initiation of treatment.

\section{Acknowledgments}

This work was supported by the grants of the National Natural Science Foundation of China (81671357), the Project of Health Department in Zhejiang Province (2014ZDA008), the National Key Basic Research Program (2016YFC1307102, 2016YFC1307104), the Public Welfare Project of Science Technology Department of Zhejiang Province (2015C33133), National Clinical Research Center for Mental Health 
Disorders (2015BAI13B02), and the Key Research Project of Zhejiang Province (2015C03040). We appreciate the patient and his guardians for their understanding. We acknowledge Dr Pornkanok Prukpitikul for her help to polish our paper.

\section{Disclosure}

The authors report no conflicts of interest in this work.

\section{References}

1. Grande I, Berk M, Birmaher B, Vieta E. Bipolar disorder. Lancet. 2016; 387(10027):1561-1572.

2. Masi G, Milone A, Veltri S, Iuliano R, Pfanner C, Pisano S. Use of quetiapine in children and adolescents. Paediatr Drugs. 2015;17(2): 125-140.

3. Jakobsen $\mathrm{KD}$, Wallach-Kildemoes $\mathrm{H}$, Bruhn $\mathrm{CH}$, et al. Adverse events in children and adolescents treated with quetiapine: an analysis of adverse drug reaction reports from the Danish Medicines Agency database. Int Clin Psychopharmacol. 2017;32(2):103-106.

4. Eyer F, Pfab R, Felgenhauer N, Strubel T, Saugel B, Zilker T. Clinical and analytical features of severe suicidal quetiapine overdoses: a retrospective cohort study. Clin Toxicol (Phila). 2011;49(9):846-853.

5. Yalug I, Tufan AE, Kayaalp L. Quetiapine may be associated with newonset seizures in patients with seizurogenic conditions. JNeuropsychiatry Clin Neurosci. 2007;19(3):341-342.

6. Dogu O, Sevim S, Kaleagasi HS. Seizures associated with quetiapine treatment. Ann Pharmacother. 2003;37(9):1224-1227.

7. Ngo A, Ciranni M, Olson KR. Acute quetiapine overdose in adults: a 5-year retrospective case series. Ann Emerg Med. 2008;52(5):541-547.

8. Young AC, Kleinschmidt KC, Wax PM. Late-onset seizures associated with quetiapine poisoning. J Med Toxicol. 2009;5(1):24-26.

9. Dev V, Raniwalla J. Quetiapine: a review of its safety in the management of schizophrenia. Drug Saf. 2000;23(4):295-307.
10. Alldredge BK. Seizure risk associated with psychotropic drugs: clinical and pharmacokinetic considerations. Neurology. 1999; 53(5 Suppl 2):S68-S75.

11. Bloechliger M, Rüegg S, Jick SS, Meier CR, Bodmer M. Antipsychotic drug use and the risk of seizures: follow-up study with a nested casecontrol analysis. CNS Drugs. 2015;29(7):591-603.

12. Lertxundi U, Hernandez R, Medrano J, Domingo-Echaburu S, García M, Aguirre C. Antipsychotics and seizures: higher risk with atypicals? Seizure. 2013;22(2):141-143.

13. Centorrino F, Price BH, Tuttle M, et al. EEG abnormalities during treatment with typical and atypical antipsychotics. Am J Psychiatry. 2002;159(1):109-115.

14. Ma L, Yang F, Zhao R, et al. Quetiapine attenuates cognitive impairment and decreases seizure susceptibility possibly through promoting myelin development in a rat model of malformations of cortical development. Brain Res. 2015;1622:443-451.

15. Klein L, Bangh S, Cole JB. Intentional recreational abuse of quetiapine compared to other second-generation antipsychotics. West $J$ Emerg Med. 2017;18(2):243-250.

16. Vimala S, Unnikrishnan P, Gautham NS. Intraoperative seizures presenting as refractory hypotension. J Neurosurg Anesthesiol. 2017; 29(2):184-185.

17. Iyer A, Appleton R. Management of reflex anoxic seizures in children. Arch Dis Child. 2013;98(9):714-717.

18. Balit CR, Isbister GK, Hackett LP, Whyte IM. Quetiapine poisoning: a case series. Ann Emerg Med. 2003;42(6):751-758.

19. Klein K, Zanger UM. Pharmacogenomics of cytochrome P450 3A4: recent progress toward the "missing heritability" problem. Front Genet. 2013;4:12.

20. Naranjo CA, Busto U, Sellers EM, et al. A method for estimating the probability of adverse drug reactions. Clin Pharmacol Ther. 1981; 30(2):239-245.
Neuropsychiatric Disease and Treatment

\section{Publish your work in this journal}

Neuropsychiatric Disease and Treatment is an international, peerreviewed journal of clinical therapeutics and pharmacology focusing on concise rapid reporting of clinical or pre-clinical studies on a range of neuropsychiatric and neurological disorders. This journal is indexed on PubMed Central, the 'PsycINFO' database and CAS,

\section{Dovepress}

and is the official journal of The International Neuropsychiatric Association (INA). The manuscript management system is completely online and includes a very quick and fair peer-review system, which is all easy to use. Visit http://www.dovepress.com/testimonials.php to read real quotes from published authors. 